Centre interuniversitaire de recherche

en économie quantitative

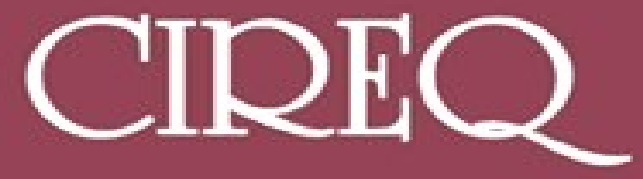

Cahier 09-2004

TIME REVERSIBILITY OF STATIONARY REGULAR FINITE STATE MARKOV CHAINS

William J. McCAUSLAND 


\title{
CIREQ
}

Le Centre interuniversitaire de recherche en économie quantitative (CIREQ) regroupe des chercheurs dans les domaines de l'économétrie, la théorie économique, la macroéconomie et les marchés financiers, l'économie du travail et l'économie de l'environnement. Ils proviennent principalement des universités de Montréal, McGill et Concordia. Le CIREQ offre un milieu dynamique de recherche en économie quantitative grâce au grand nombre d'activités qu'il organise (séminaires, ateliers, colloques) et de collaborateurs qu'il reçoit chaque année.

The Center for Interuniversity Research in Quantitative Economics (CIREQ) regroups researchers in the fields of econometrics, economic theory, macroeconomics and financial markets, labor economics, and environmental economics. They come mainly from the Université de Montréal, McGill University and Concordia University. CIREQ offers a dynamic environment of research in quantitative economics thanks to the large number of activities that it organizes (seminars, workshops, conferences) and to the visitors it receives every year.

\section{Cahier 09-2004}

TIME REVERSIBILITY OF STATIONARY REGULAR FINITE STATE MARKOV CHAINS

\author{
William J. McCAUSLAND
}

téléphone : (514) 343-6557 télécopieur : (514) 343-5831 cireq@umontreal.ca http://www.cireq.umontreal.ca

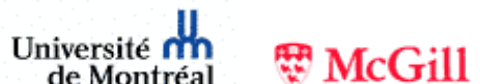
Concordia 
Ce cahier a également été publié par le Département de sciences économiques de l'Université de Montréal sous le numéro 2004-07.

This working paper was also published by the Département de sciences économiques of the Université de Montréal, under number 2004-07.

Dépôt légal, Bibliothèque nationale du Canada, 2004, ISSN 0821-4441

Dépôt légal, Bibliothèque nationale du Québec, 2004, ISBN 2-89382-486-2 


\title{
Time Reversibility of Stationary Regular Finite State Markov Chains
}

\author{
William J. McCausland* \\ Département de sciences économiques, Université de Montréal, \\ CIREQ and CIRANO \\ www.cirano.qc.ca/ mccauslw
}

May 27, 2004

\begin{abstract}
We propose an alternate parameterization of stationary regular finite-state Markov chains, and a decomposition of the parameter into time reversible and time irreversible parts. We demonstrate some useful properties of the decomposition, and propose an index for a certain type of time irreversibility. Two empirical examples illustrate the use of the proposed parameter, decomposition and index. One involves observed states; the other, latent states.
\end{abstract}

Key Words: Finite-state Markov chains, Time reversibility, Bayesian inference, Hidden Markov Models

\section{Résumé}

Nous proposons une paramétrisation alternative des chaînes markoviennes stationnaires et régulières à états finis, ainsi qu'une décomposition du paramètre en une partie réversible et une partie irréversible. Nous démontrons certaines propriétés utiles de cette décomposition et proposons une mesure pour un type particulier d'irréversibilité temporelle. Deux exemples empiriques illustrent l'utilisation du paramètre proposé, sa décomposition et la mesure. Le premier traite d'états observables, alors que le second traite d'états latents.

Mots clés: chaînes markoviennes à états finis, réversibilité temporelle, inférence bayesienne, modeles de Markov cachés

${ }^{*}$ I thank FQRSC (Fonds québécois de la recherche sur la société et la culture) for financial support, Simon Leblond for excellent research assistance, John Geweke for helpful comments, and Andrew Eckert for providing me with data. Computations reported in this paper were undertaken using the Bayesian Analysis, Computation and Communication (BACC) software described in Geweke (1999) and McCausland (2004). Additional software for computations reported in this paper is available from the author. I alone am responsible for errors. 


\section{Introduction}

Intuitively, a random process is time reversible if the statistical properties of the process are the same as those of the same series running backwards through time. For discrete-time processes, a formal definition is the following.

Definition 1.1 A discrete-time random process $\left\{x_{t}\right\}$ is time reversible if for every positive integer $K$ and all integers $t$ and $\tau$, the distributions of $\left(x_{t}, x_{t+1}, \ldots, x_{t+K}\right)$ and $\left(x_{\tau-t}, x_{\tau-t-1}, \ldots, x_{\tau-t-K}\right)$ are identical.

Several papers in economics find evidence of time irreversibility in observed time series. In the large literature on business cycle fluctuations, many articles document a tendency for downswings to be faster than upswings, an example of what is called in the literature "steepness". Ramsey and Rothman (1996) survey much of this literature, and find evidence of time irreversibility in many macroeconomic variables. Chen and Kuan (2001) find that stock index returns are time irreversible, and Fong (2003) finds that shocks to trading volume and volatility of stock returns are time irreversible. Noel (2003) analyses retail gasoline markets in 19 Canadian cities and finds strong evidence, in some but not all markets, of cycles where prices tend to rise sharply and decline gradually.

Although time reversibility is quite restrictive, there are many examples of common statistical models for discrete time processes that impose it. Time reversible processes include exchangeable processes, univariate stationary Gaussian processes and time discretized stationary univariate diffusions. The latter two are often used as models for macroeconomic variables and asset returns, respectively.

Allowing time irreversibility is not only important for constructing more realistic statistical models. Time reversibility may be of economic interest in itself. Maskin and Tirole (1988) discuss a dynamic game in which two firms competing in an output market choose prices in each period. One set of equilibria feature what the authors call "Edgeworth cycles", in which large price jumps are followed by more gradual falls in price as the two firms repeatedly undercut one another. Noel (2003) explicitly draws the connection between cycles in gasoline prices and Edgeworth cycles, and shows that the market penetration of independent firms helps predict the presence or absence of cycles in a market, in a way that is consistent with an extension by Eckert (2003) of the theory of Maskin and Tirole. 
In markets where participants simultaneously observe noisy value-relevant signals and learn about the signal through time, price volatility and trading volume may feature rapid growth and slow decay. Peng and Xiong (2003) propose a model with information processing capacity constraints (see also Sims (2001)) that features not only the clustering and long memory of volatility (their objective) but also cycles of volatility with sharp increases and gradual decreases.

Decisions by economic agents, such as the capital investment decisions of firms, may be more easily made than reversed, leading to time irreversible processes for choice variables.

Tests for time reversibility have been proposed by various authors. Ramsey and Rothman (1996) introduce a time domain test and Hinich and Rothman (1998) propose a frequency domain test. Robinson (1991) describes an entropy based test that can be used to test time reversibility. Chen, Chou and Kuan (2000) introduce a class of tests based on characteristic functions that do not require the existence of any moments.

Stationary regular finite-state Markov chains can be reversible or not. When they are parameterized as described in this paper, time reversibility is easy to check and the degree and nature of time irreversibility is transparent. When the number of states is greater than two, imposition of time reversibility amounts to a reduction in the dimension of the parameter space. This parsimony may be useful in some applications. Incorporating latent Markov chains is an easy way of generating models that can feature time reversibility or not, depending on values of the parameters.

Section 2 reviews relevant results about stationary regular finite-state Markov chains and proposes an alternate parameterization. Section 3 introduces a decomposition of the parameter into what we will call its time reversible and time irreversible parts, and proposes an index for a certain type of time irreversibility. Section 4 presents results from two empirical applications. Section 5 concludes.

\section{An Alternate Parameterization}

A stationary regular finite-state Markov chain $\left\{s_{t}\right\}$ is usually parameterized by its Markov transition matrix $P$, which gives the conditional probabilities $\operatorname{Pr}\left[s_{t}=j \mid s_{t-1}=i\right]$. We first review some results on stationary regular finitestate Markov chains. We then introduce an alternate parameterization of 
these chains by the matrix $\Pi$ of joint probabilities $\operatorname{Pr}\left[s_{t-1}=i, s_{t}=j\right]$. We show that symmetry of this matrix is equivalent to the time reversibility of the chain.

\subsection{A Conventional Parameterization}

Let $\left\{s_{t}\right\}$ be a Markov chain with finite state space $\{1, \ldots, m\}$. Let $P$ be its $m \times m$ transition matrix. That is, for all $i, j \in\{1, \ldots, m\}$,

$$
P_{i j}=\operatorname{Pr}\left[s_{t}=j \mid s_{t-1}=i\right] \text {. }
$$

We review the following important and well known results. See Iosifescu (1980), and especially Theorem 1.9, Proposition 4.1 and Theorems 4.2 and 4.4 .

1. $\left\{s_{t}\right\}$ is regular ${ }^{1}$ if and only if $P$ is regular ${ }^{2}$

2. If $\left\{s_{t}\right\}$ is regular, then

(a) there exists a unique $1 \times m$ row-stochastic ${ }^{3}$ vector $\pi$, which we will call the stationary distribution of $\left\{s_{t}\right\}$, such that $\pi P=\pi$,

(b) $\pi>0$, and

(c) if $\left\{s_{t}\right\}$ is stationary, then for all $i \in\{1, \ldots, m\}$ and all $t, \pi_{i}=$ $\operatorname{Pr}\left[s_{t}=i\right]$.

The results imply that a stationary regular finite-state Markov chain is fully described by its Markov transition matrix, and that the following parameter set indexes the stationary regular $m$-state Markov chains.

$$
\mathbf{P} \equiv\left\{P \in \mathbb{R}^{m \times m}: P \text { is row-stochastic and regular }\right\} .
$$

\footnotetext{
${ }^{1} \mathrm{~A}$ Markov chain is regular is it is irreducible and aperiodic.

${ }^{2} \mathrm{~A}$ Markov transition matrix $P$ is regular if there exists an integer $n>0$ such that for all states $i$ and $j,\left(P^{n}\right)_{i j}>0$.

${ }^{3}$ A real $m \times n$ matrix (or vector) $P$ is row-stochastic if for all $i, j \in\{1, \ldots, m\}$, $\sum_{k=1}^{m} P_{i k}=1$ and $P_{i j} \geq 0$.
} 


\subsection{An Alternate Parameterization}

For the purposes of studying time reversibility and time irreversibility of stationary regular finite-state Markov chains, it is convenient to consider an alternate parameterization, one which gives joint state probabilities rather than conditional state probabilities.

Definition 2.1 Let $\left\{s_{t}\right\}$ be a stationary regular finite-state Markov chain. Define the joint probability matrix $\Pi$ of $\left\{s_{t}\right\}$ as the $m \times m$ matrix such that for all $i, j \in\{1, \ldots, m\}, \Pi_{i j} \equiv \operatorname{Pr}\left[s_{t-1}=i, s_{t}=j\right]$.

We introduce three definitions that will be useful for describing the properties of $\Pi$. We say that an $m \times m$ matrix $A$ is balanced if for all $i \in\{1, \ldots, m\}$,

$$
\sum_{j=1}^{m} A_{i j}=\sum_{j=1}^{m} A_{j i}
$$

matrix-stochastic if for all $i, j \in\{1, \ldots, m\}, A_{i j} \geq 0$ and

$$
\sum_{i=1}^{m} \sum_{j=1}^{m} A_{i j}=1,
$$

and regular ${ }^{4}$ if there exists an integer $n>0$ such that for all $i, j \in\{1, \ldots, m\}$, $\left(A^{n}\right)_{i j}>0$.

The following result demonstrates that for a stationary regular finite-state Markov chain, the joint probability matrix $\Pi$ can be calculated from the Markov transition matrix $P$ and vice versa, that the stationary distribution $\pi$ can easily be recovered from $\Pi$ and that $\Pi$ must be balanced, matrix stochastic and regular.

Result 2.1 Let $\left\{s_{t}\right\}$ be a stationary regular finite-state Markov chain, $P$ be its Markov transition matrix, $\pi$ be its stationary distribution, $\Pi$ be its joint probability matrix and $D$ be the diagonal matrix with $D_{i i}=\pi_{i}$ for all $i \in\{1, \ldots, m\}$. Then

$$
\text { 1. } \Pi=D P \text {, }
$$

\footnotetext{
${ }^{4}$ This is a generalization of the definition of regularity of Markov transition matrices to square matrices.
} 
2. $D$ is non-singular and $P=D^{-1} \Pi$,

3. $\pi=\iota^{\prime} \Pi=(\Pi \iota)^{\prime}$, where $\iota$ is an $m \times 1$ vector of ones,

4. $\Pi$ is balanced, matrix stochastic and regular.

Proof.

1. For all $i, j \in\{1, \ldots, m\}$,

$$
\Pi_{i j}=\operatorname{Pr}\left[s_{t-1}=i, s_{t}=j\right]=\operatorname{Pr}\left[s_{t}=i\right] \cdot \operatorname{Pr}\left[s_{t}=j \mid s_{t-1}=i\right]=\pi_{i} P_{i j},
$$

2. Since $\pi$ is positive, $D$ is non-singular and thus invertible. Together with the previous result, we have $P=D^{-1} \Pi$.

3. Stationary gives, for all $i \in\{1, \ldots, m\}$,

$$
\pi_{i}=\operatorname{Pr}\left[s_{t}=i\right]=\sum_{j=1}^{m} \operatorname{Pr}\left[s_{t-1}=j, s_{t}=i\right]=\sum_{j=1}^{m} \Pi_{j i}
$$

and

$$
\pi_{i}=\operatorname{Pr}\left[s_{t-1}=i\right]=\sum_{j=1}^{m} \operatorname{Pr}\left[s_{t-1}=i, s_{t}=j\right]=\sum_{j=1}^{m} \Pi_{i j} .
$$

4. The previous result implies that $\Pi$ is balanced. We have

$$
\sum_{i=1}^{m} \sum_{j=1}^{m} \Pi_{i j}=\sum_{i=1}^{m} \sum_{j=1}^{m} \operatorname{Pr}\left[s_{t-1}=i, s_{t}=j\right]=1,
$$

and for all $i, j \in\{1, \ldots, m\}$,

$$
\Pi_{i j}=\operatorname{Pr}\left[s_{t-1}=i, s_{t}=j\right] \geq 0 .
$$

Therefore $\Pi$ is matrix-stochastic. Given that the elements of $P$ and $\Pi$ are non-negative, regularity depends only on which of these elements are zero. Since for all $i, j \in\{1, \ldots, m\}, P_{i j}>0 \Longleftrightarrow \Pi_{i j}>0$, regularity of $P$ implies regularity of $\Pi$.

The next result demonstrates that the properties of balance, matrixstochasticity and regularity are exhaustive. 
Result 2.2 Let $m \times m$ matrix $\Pi$ be balanced, matrix-stochastic and regular. Then $\Pi$ is the joint probability matrix for a stationary regular m-state Markov chain.

Proof. Let $\pi \equiv \iota^{\prime} \Pi$, which is equal to $(\Pi \iota)^{\prime}$ by balance. Let $D$ be the diagonal matrix with $D_{i i}=\pi_{i}$ for all $i \in\{1, \ldots, m\}$. Regularity of $\Pi$ rules out a row or column of zeros, so $\pi>0$ and $D$ is non-singular. Now let $P \equiv D^{-1} \Pi$.

Matrix stochasticity of $\Pi$ implies that $P \iota=D^{-1} \Pi \iota=D^{-1} \pi^{\prime}=\iota$, that $\pi \iota=\iota^{\prime} \Pi \iota=1$, and that for all $i, j \in\{1, \ldots, m\}, P_{i j} \geq 0$. Therefore $\pi$ and $P$ are row-stochastic.

Since for all $i, j \in\{1, \ldots, m\}, \Pi_{i j}>0 \Longleftrightarrow P_{i j}>0$, regularity of $\Pi$ implies regularity of $P$.

Now let $\left\{s_{t}\right\}$ be a stationary $m$-state Markov chain whose Markov transition matrix is $P$. Regularity of $P$ implies regularity of $\left\{s_{t}\right\}$. Since $\pi P=$ $\iota^{\prime} D P=\iota^{\prime} \Pi=\pi$ and $P$ is row-stochastic, $\pi$ is the stationary distribution of $P$. Since $\Pi=D P, \Pi$ is the joint probability matrix of regular stationary finite-state Markov chain $\left\{s_{t}\right\}$.

We have now established the following parameter set as an alternative to $\mathbf{P}$ for stationary regular finite-state Markov chains:

$$
\Pi=\left\{\Pi \in \mathbb{R}^{m \times m}: \Pi \text { is balanced, matrix-stochastic, and regular }\right\} \text {. }
$$

An important advantage of the $\Pi$ parameterization lies in the following result, which states that the reversibility of a stationary regular finite Markov chain is equivalent to the symmetry of its joint probability matrix, a condition easy to check.

Result 2.3 Let $\left\{s_{t}\right\}$ be a stationary regular finite Markov chain, and let $\Pi$ be its joint probability matrix. Then $\left\{s_{t}\right\}$ is time reversible if and only if $\Pi$ is symmetric.

Proof. Suppose $\left\{s_{t}\right\}$ is time reversible. Taking $K=2$ and $\tau=2 t+1$ in the definition of time reversibility, we have, for all $i, j \in\{1, \ldots, m\}$,

$$
\Pi_{i j}=\operatorname{Pr}\left[s_{t-1}=i, s_{t}=j\right]=\operatorname{Pr}\left[s_{t}=i, s_{t-1}=j\right]=\operatorname{Pr}\left[s_{t-1}=j, s_{t}=i\right]=\Pi_{j i},
$$

so $\Pi$ is symmetric. 
Now suppose that $\Pi$ is symmetric. Let positive integer $K$ and state sequence $i_{0}, i_{1}, \ldots, i_{K} \in\{1, \ldots, m\}$ be arbitrary. Then

$$
\begin{aligned}
\operatorname{Pr}\left[s_{t}=i_{0}, \ldots, s_{t+K}=i_{K}\right] & =\pi_{i_{0}} \prod_{k=1}^{K} P_{i_{k-1} i_{k}}=\pi_{i_{0}} \prod_{k=1}^{K} \pi_{i_{k-1}}^{-1} \prod_{i_{k-1} i_{k}} \\
& =\left(\prod_{k=1}^{K-1} \pi_{i_{k}}^{-1}\right) \prod_{k=1}^{K} \Pi_{i_{k-1} i_{k}}
\end{aligned}
$$

and

$$
\begin{aligned}
\operatorname{Pr}\left[s_{\tau-t}=i_{0}, \ldots, s_{\tau-t-K}=i_{K}\right] & =\operatorname{Pr}\left[s_{\tau-t-K}=i_{K}, \ldots, s_{\tau-t}=i_{0}\right] \\
& =\pi_{i_{K}} \prod_{k=K}^{1} P_{i_{k} i_{k-1}}=\pi_{i_{K}} \prod_{k=K}^{1} \pi_{i_{k}}^{-1} \Pi_{i_{k} i_{k-1}} \\
& =\left(\prod_{k=1}^{K-1} \pi_{i_{k}}^{-1}\right) \prod_{k=1}^{K} \Pi_{i_{k-1} i_{k}} \\
& =\operatorname{Pr}\left[s_{t}=i_{0}, \ldots, s_{t+K}=i_{K}\right],
\end{aligned}
$$

so $\left\{s_{t}\right\}$ is time reversible.

A second advantage of the $\Pi$ parameter over the $P$ parameter is the transparency of the stationary distribution $\pi$. While calculating $\pi$ from $P$ involves finding a left eigenvector for the unit eigenvalue, computing $\pi$ from $\Pi$ is a linear operation: $\pi=\iota^{\prime} \Pi=\iota^{\prime} \Pi^{\prime}$. It is easier to compute $P$ and $\pi$ from $\Pi$ than $\Pi$ and $\pi$ from $P$.

\subsection{A Graphical Representation of $\Pi$}

We construct a directed graph with the same information as $\Pi$. The graph has $m$ vertices, labelled 1 through $m$, each representing one of the states of the underlying Markov chain. For all vertices $i, j \in\{1, \ldots, m\}$, there is directed edge of weight $\Pi_{i j}$ from $i$ to $j$. We will call $\Pi_{i j}$ the flow from state $i$ to state $j, \Pi_{i j}+\Pi_{j i}$ the total flow between states $i$ and $j, \sum_{k=1}^{m} \Pi_{i k}$ the outflow from state $i$ and $\sum_{k=1}^{m} \prod_{k i}$ the inflow to state $i$.

Figure 1 shows a $3 \times 3$ joint probability matrix and the corresponding graph. Line thicknesses are proportional to the corresponding elements of $\Pi$. The balance condition on $\Pi$ is equivalent to the property that the inflow to a state equals the outflow from it. Balance is intimately related to stationarity, 
Figure 1: A joint probability matrix $\Pi$ and a graphical representation

$$
\Pi=\frac{1}{20}\left[\begin{array}{lll}
0 & 2 & 3 \\
1 & 1 & 3 \\
4 & 2 & 4
\end{array}\right]
$$

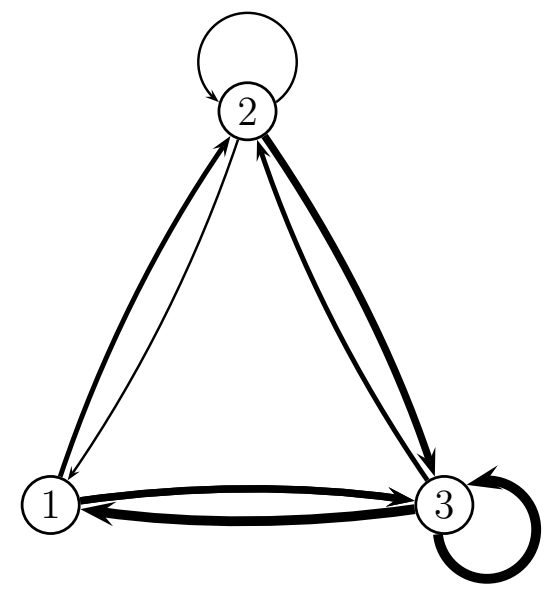

since the outflow from a state $i$ gives the marginal probability $\operatorname{Pr}\left[s_{t-1}=i\right]$ and the inflow to $i$ gives the marginal probability $\operatorname{Pr}\left[s_{t}=i\right]$.

We have seen that reversibility of $\left\{s_{t}\right\}$ is equivalent to the symmetry of $\Pi$. This is equivalent in turn to the property that for all states $i$ and $j$, the flow from $i$ to $j$ is equal to the flow from $j$ to $i$. This condition is known as detailed balance.

Notice that for $m=2$, balance implies detailed balance or equivalently, symmetry of $\Pi$. Thus all stationary regular two-state Markov chains are time reversible.

\section{Time Reversibility and a Decomposition}

We describe a decomposition of the joint probability matrix $\Pi$. We demonstrate several properties of the decomposition that are useful for testing for time reversibility and for characterizing time irreversibility. We introduce an index for a certain type of time irreversibility in applications where states have a natural order. 


\subsection{A Decomposition}

Any $\Pi \in \Pi$ can be decomposed as $\Pi=X+L$, where

$$
X \equiv\left(\Pi+\Pi^{\prime}\right) / 2 \quad \text { and } \quad L \equiv\left(\Pi-\Pi^{\prime}\right) / 2
$$

Anticipating future results, we will call $X$ the time reversible part of $\Pi$ and $L$ the time irreversible part of $\Pi$. The symbols $X$ and $L$ are chosen because the symmetry and asymmetry of these letters resemble the symmetry and asymmetry of the matrices they represent.

The first important result is that the time reversible part of a joint probability matrix is the joint probability matrix for a time reversible stationary regular finite Markov chain.

Result 3.1 Suppose $\Pi \in \Pi$ and let $X=\left(\Pi+\Pi^{\prime}\right) / 2$. Then $X \in \Pi$ and $X^{\prime}=X$.

Proof. That $X$ is balanced and matrix-stochastic is obvious. For all $i, j \in$ $\{1, \ldots, m\}, X_{i j} \geq 0$ and $X_{i j}>0$ whenever $\Pi_{i j}>0$, so $X$ must be regular. $X^{\prime}=X$ is obvious.

The next result summarizes properties that a joint probability matrix $\Pi$ and its reversible part $X$ have in common: the chains they govern have the same stationary distribution, the same state persistence $\operatorname{Pr}\left[s_{t}=i \mid s_{t-1}=i\right]=$ $\Pi_{i i} / \pi_{i}$ in every state $i$, and the same total flow $\Pi_{i j}+\Pi_{j i}$ between any states $i$ and $j$.

Result 3.2 Suppose $\Pi \in \Pi$ and let $X=\left(\Pi+\Pi^{\prime}\right) / 2$ and $L=\left(\Pi-\Pi^{\prime}\right) / 2$. Let $\pi$ be the stationary distribution of the chain whose joint probability matrix is $\Pi$. Let $P$ and $P^{X}$ be the Markov transition matrices associated with $\Pi$ and $X$. Then

$$
\begin{aligned}
& \text { 1. } \pi P^{X}=\pi P=\pi \text {, } \\
& \text { 2. for all } i \in\{1, \ldots, m\}, P_{i i}^{X}=P_{i i} \text {, and } \\
& \text { 3. } X+X^{\prime}=\Pi+\Pi^{\prime} \text {. }
\end{aligned}
$$

Proof. For all $i \in\{1, \ldots, m\}, \sum_{j=1}^{m} \Pi_{i j}=\sum_{j=1}^{m} X_{i j}=\pi_{i}$, so $\pi$ gives the stationary distribution for both the $\Pi$ and the $X$ chains, and therefore $\pi P^{X}=$ $\pi P=\pi$. In addition, for all $i \in\{1, \ldots, m\}, \Pi_{i i}=X_{i i}$ and therefore $P_{i i}=$ $\Pi_{i i} / \pi_{i}=X_{i i} / \pi_{i}=P_{i i}^{X} . X+X^{\prime}=\Pi+\Pi^{\prime}$ is obvious. 
We now define the subset $\mathbf{X}$ of $\boldsymbol{\Pi}$ of joint probability matrices for time reversible stationary regular finite state Markov chains.

$$
\mathbf{X} \equiv\{\Pi \in \Pi: \Pi \text { is symmetric }\}
$$

The next result is that the time reversibility of a stationary regular finite Markov chain is equivalent to its joint probability matrix $\Pi$ being equal to its reversible part and equivalent to its irreversible part being zero.

Result 3.3 Suppose $\Pi \in \Pi$ and let $X=\left(\Pi+\Pi^{\prime}\right) / 2$ and $L=\left(\Pi-\Pi^{\prime}\right) / 2$. Then $\Pi \in \mathbf{X} \Leftrightarrow X=\Pi \Leftrightarrow L=0$.

Proof. Obvious.

\subsection{A Graphical Representation of $X$ and $L$}

Let $X$ and $L$ be the time reversible and time irreversible parts of a joint probability matrix $\Pi$. We can construct an undirected graph with the same information as $X$. For all vertices $i, j \in\{1, \ldots, m\}$, there is an undirected edge of weight $X_{i j}=X_{j i}$ from $i$ to $j$. Figure 2 shows the time reversible part $X$ of the joint probability matrix $\Pi$ of Figure 1, and the corresponding graph. Line thicknesses are proportional to the corresponding elements of $X$.

We can construct a directed graph with the same information as $L$. For all vertices $i, j \in\{1, \ldots, m\}$, there is an directed edge of weight $\left|L_{i j}\right|=\left|L_{j i}\right|$ between $i$ and $j$. If $L_{i j}$ is positive, the direction is from $i$ to $j$; if negative, from $j$ to $i$. Figure 3 shows the time irreversible part $L$ of the joint probability matrix $\Pi$ of Figure 1, and the corresponding graph. Line thicknesses are proportional to the corresponding elements of $L$.

Suppose we have a stationary regular finite state Markov chain with joint probability matrix $\Pi$. It is well known that the time reversed chain is also stationary, regular and Markov. Let $\Pi_{r}$ be its joint probability matrix and and let $X_{r}=\left(\Pi_{r}+\Pi_{r}^{\prime}\right) / 2$ and $L_{r}=\left(\Pi_{r}-\Pi_{r}^{\prime}\right) / 2$. It is easy to see that $\Pi_{r}=\Pi^{\prime}, X_{r}=X$ and $L_{r}=L^{\prime}=-L$. The graphical representation of the time reversed chain will have the same edges as that of the original chain, with all directions reversed.

\subsection{An Index of Time Reversibility}

In many applications, including the two examples in this paper, states have a natural order. We assume states are labelled in a manner consistent with 
Figure 2: Matrix $X$ and a graphical representation

$$
X=\frac{1}{40}\left[\begin{array}{lll}
0 & 3 & 7 \\
3 & 2 & 5 \\
7 & 5 & 8
\end{array}\right]
$$

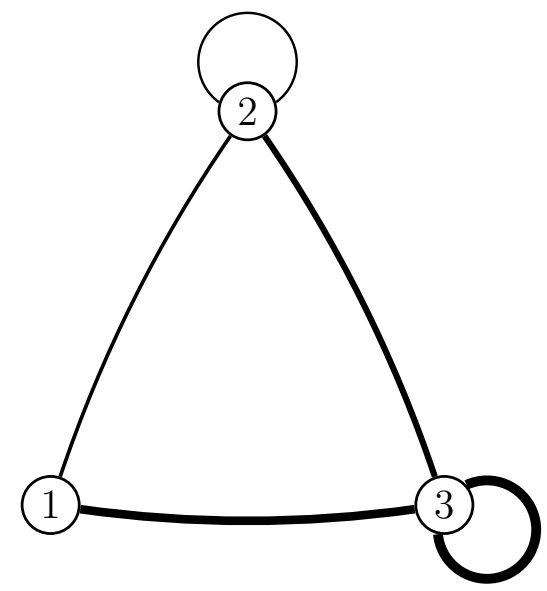

this order. So, for example, state $m$ is the highest and state 1 is the lowest. In this section, we describe an index for a certain type of time irreversibility, applicable to chains with ordered states. It is designed to capture the degree to which state transitions to higher states tend to be smaller and more frequent than those to lower states. The index can be negative, in which case jumps are larger and less frequent than falls. Consider, by way of example, the $\Pi$ matrix in Figure 1. The sum of the elements above the diagonal $(8 / 20)$ gives the probability of a jump to a higher state from one observation to the next. The sum of the elements below the diagonal $(7 / 20)$, giving the probability of a fall, is smaller. However, the conditional expectation of the size of a jump, given a jump, is

$$
\Pi_{12}+2 \Pi_{13}+\Pi_{23} /\left(\Pi_{12}+\Pi_{13}+\Pi_{23}\right)=11 / 8,
$$

while that of the size of a fall, given a fall, is

$$
\Pi_{21}+2 \Pi_{31}+\Pi_{32} /\left(\Pi_{21}+\Pi_{31}+\Pi_{32}\right)=11 / 7,
$$

which is larger.

Take an interior state $i \in\{2, \ldots, m-1\}$ and consider the net outflow $C_{i} \equiv \sum_{j>i} L_{i j}$ from state $i$ to higher states. Balance implies that $C_{i}$ is 
Figure 3: Matrix $L$ and a graphical representation

$$
L=\frac{1}{40}\left[\begin{array}{ccc}
0 & 1 & -1 \\
-1 & 0 & 1 \\
1 & -1 & 0
\end{array}\right]
$$

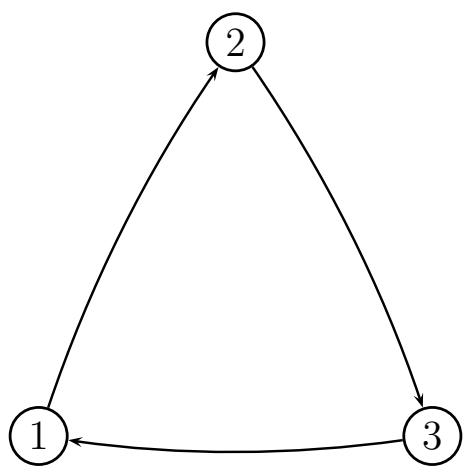

also the net inflow $\sum_{j<i}-L_{i j}$ to $i$ from lower states. Balance also implies that this flow must be offset by a net flow $C_{i}$ from states higher than $i$ directly to states lower than $i$. We will call $C_{i}$ the circulation through $i$ and $C \equiv \sum_{i=2}^{m-1} C_{i}=\sum_{j>i} L_{i j}$ the total circulation.

For the $L$ matrix in Figure 3, corresponding to the $\Pi$ matrix in Figure 1, we calculate the circulation $C_{2}$ through state 2 and the total circulation $C$ to be $L_{23}=1 / 40$.

Clearly, time reversibility implies $C=0$, but the converse is not true for $m>3$. Figure 4 represents on the left a non-zero $L$ matrix for which $C=0$. Negative circulation through state 2 is offset by positive circulation through state 3 . On the right is a representation of an $L$ matrix whose total circulation is positive: all interior states have positive circulation.

We see that $C$ is a useful index for a particular type of time reversibility where circulation through a state tends to be in the same direction across states.

Recalling examples from the introduction, we see that the tendancy for gasoline price increases to be sharper and less frequent than decreases is similar to negative circulation, and that the tendancy for economic downturns to be faster than upturns is similar to positive circulation. 
Figure 4: Time reversibility with and without zero total circulation
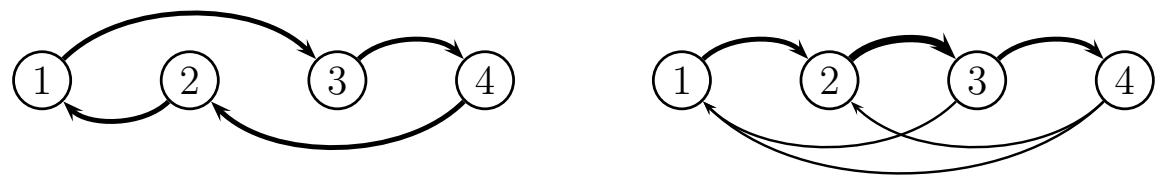

\section{Empirical Examples}

The first empirical example investigates the time irreversibility of gasoline price mark-ups. We have 267 weekly observations of retail price $r_{t}$ and wholesale price $w_{t}$ for gasoline from November 27, 1989 to September 25, 1994. $r_{t}$ is an average for a sample of gasoline stations in Windsor, Ontario, Canada. $w_{t}$ is the price charged for large scale purchases of unbranded gasoline at the terminal in Toronto, Ontario. The data, collected by the government of Ontario, are the same as those used in Eckert (2002).

We divide the mark-up $r_{t} / w_{t}$ into 6 bins according to Table 1 and model the evolution of the mark-up bin $s_{t}$ as a stationary regular 6-state Markov chain.

\begin{tabular}{|c|c|}
\hline State & Range \\
\hline 1 & $r_{t} / w_{t}<1.0$ \\
\hline 2 & $1.0 \leq r_{t} / w_{t}<1.1$ \\
\hline 3 & $1.1 \leq r_{t} / w_{t}<1.2$ \\
\hline 4 & $1.2 \leq r_{t} / w_{t}<1.3$ \\
\hline 5 & $1.3 \leq r_{t} / w_{t}<1.4$ \\
\hline 6 & $1.4 \leq r_{t} / w_{t}$ \\
\hline
\end{tabular}

We choose a prior on $P$ with independent rows, each having a Dirichlet distribution. The Dirichlet parameter associated with $P_{i j}$ is 2 if $i=j$ and 
1 otherwise. We note that $P$ is regular with probability one. The chain is irreversible with probability one, but since the density of $P$ is invariant to state relabelling, the prior is neutral with respect to the direction of cycles in the $L$ matrix.

We use the BACC software, described in Geweke (1999) and McCausland (2004), to generate a posterior sample of 100,000 draws of $P$ and then construct posterior samples for $\pi, \Pi, X$ and $L$. Tables 2 and 3 show the posterior mean and standard deviation for each element of the matrices $P$, $\pi, \Pi, X$ and $L$. Only the upper triangle of $X$ and $L$ are shown, the elements below the diagonal being redundant $\left(X_{i j}=X_{j i}\right.$ and $\left.L_{i j}=-L_{j i}\right)$.

Table 4 shows the posterior mean and standard deviation of the circulation through states 2, 3, 4 and 5, and the total circulation. Figure 5 displays a histogram for the posterior sample of total circulation. There is strong evidence of negative total circulation and negative circulation through state 3 , and moderate evidence of negative circulation through states 2,4 and 5 .

This evidence is consistent with previous observations that prices tend to rise sharply and decline gradually. It also suggests that total circulation measures a type of time reversibility that is empirically relevant.

The second empirical example investigates time irreversibility of investment growth in the U.S.A. We have 205 quarterly observations, from the first quarter of 1947 to the first quarter of 2004, of real gross private domestic investment ${ }^{5} I_{t}$, We construct the series $g_{t} \equiv \log \left(I_{t} / I_{t-1}\right)$ of investment growth. This is the same series used in Clements and Krolzig (2003) in their investigation of business cycle asymmetries, updated to the first quarter of 2004.

We use one of the models in Clements and Krolzig (2003), originally described in Hamilton (1989), for $g_{t}$. There is a latent stationary regular Markov state sequence $\left\{s_{t}\right\}$ on the state set $\{1, \ldots, m\}$, with transition matrix $P$. The process $\left\{g_{t}\right\}$, conditional on $\left\{s_{t}\right\}$, is given by

$$
\begin{aligned}
g_{t}-\mu_{s_{t}} & =\sum_{i=1}^{p} \phi_{i}\left(g_{t-i}-\mu_{s_{t-1}}\right)+\epsilon_{t} \\
\epsilon_{t} & \sim \text { i.i.d. } N\left(0, h^{-1}\right) .
\end{aligned}
$$

\footnotetext{
${ }^{5} I_{t}$ is in billions of chained 2000 dollars, seasonally adjusted, at an annual rate. The source is the FRED II (Federal Reserve Economic Data) database (www.stls.ftb.org/fred/data/gdp.html) and the series ID is GPDIC96.
} 


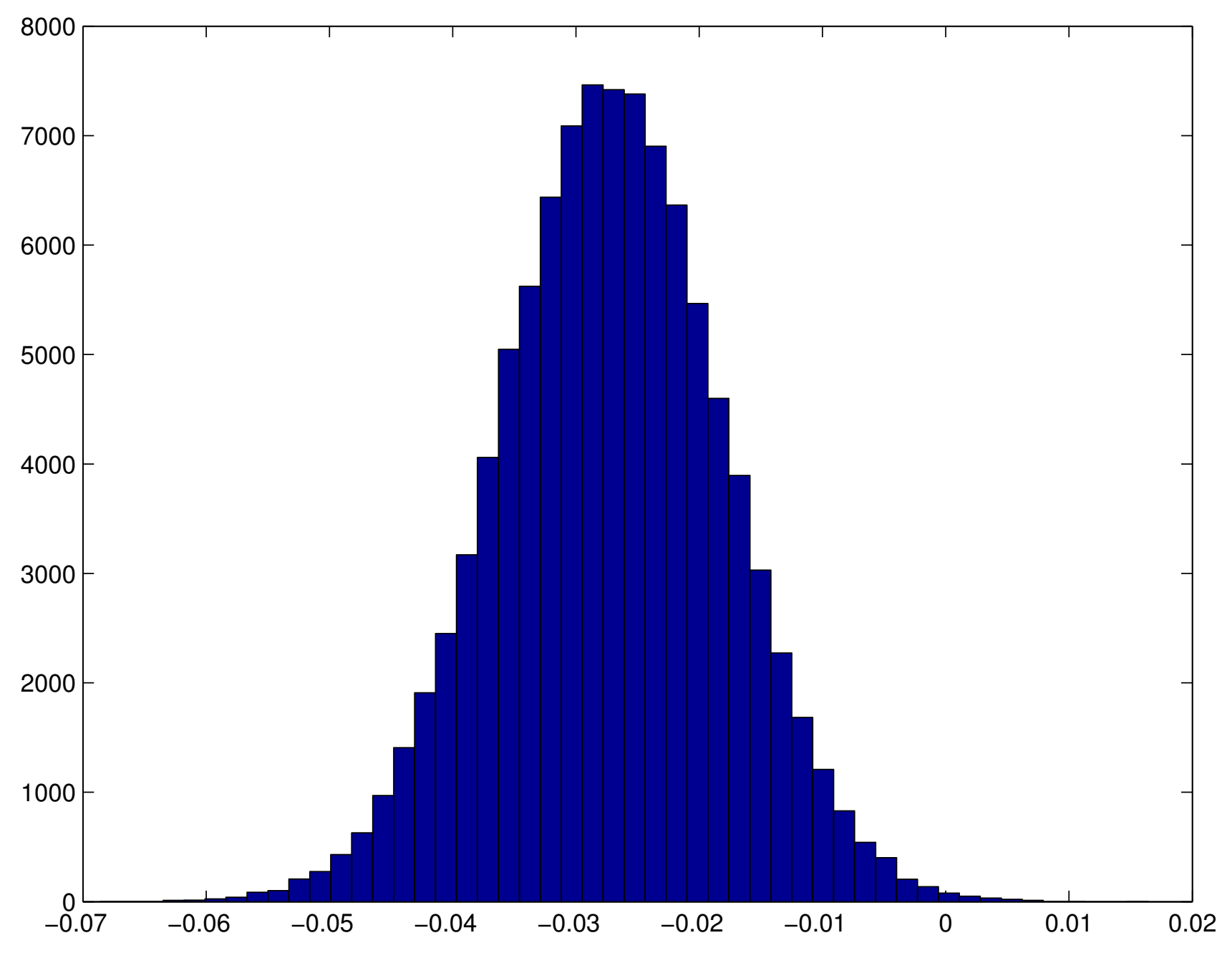

Figure 5: Posterior histogram of total circulation for gasoline mark-up example 
The quantities $\mu \equiv\left(\mu_{1}, \ldots \mu_{m}\right), h, P$ and $\phi \equiv\left(\phi_{1}, \ldots, \phi_{p}\right)$ are unknown parameters.

Following Clements and Krolzig, we set $m=3$ and $p=4$. We complete the model with a prior on $\mu, h, P$ and $\phi$ where the parameters are independent. $\mu$ has a truncated multivariate normal distribution. The untruncated distribution has independent $\mu_{2}, \mu_{3}-\mu_{2}$ and $\mu_{2}-\mu_{1}$, with means ${ }^{6} 0.01,0.04$ and 0.04 and standard deviations $0.02,0.04$ and 0.04 . Truncation is to the set where $\mu_{1}<\mu_{2}<\mu_{3}$. The prior for $h$ has $0.0004 \cdot h \sim \chi^{2}(1)$. The rows of $P$ are independent, with the following Dirichlet distributions:

$$
\begin{aligned}
& \left(P_{11}, P_{12}, P_{13}\right) \sim \operatorname{Di}(2,2,1), \\
& \left(P_{21}, P_{22}, P_{23}\right) \sim \operatorname{Di}(1,3,1), \\
& \left(P_{31}, P_{32}, P_{33}\right) \sim \operatorname{Di}(1,2,2) .
\end{aligned}
$$

$\phi$ has a truncated multivariate normal distribution. The untruncated distribution has independent $\phi_{i}$, with $\phi_{1} \sim N(0,1)$ and $\phi_{i} \sim N(0.25)$ for $i \in\{2,3,4\}$. Truncation is to the stationary region.

We note that $y_{t}-\mu_{s t}$ is stationary and Gaussian and therefore time reversible, so any time irreversibility must come in through $s_{t}$. We also note that the density for $P$ is invariant to the relabelling of states 1 and 3 , so that the prior is neutral with respect to the direction of cycles in the $L$ matrix.

Using the BACC software, we generate a posterior sample of 100,000 draws of all parameters. We then construct posterior samples for $\pi, \Pi, X$ and $L$. Table 5 shows the posterior mean and standard deviation of the elements of $P, \pi, \Pi, X$ and $L$. Figure 6 displays a histogram for the posterior sample of total circulation. There is moderate evidence of negative total circulation.

\section{Conclusions}

We have introduced the parameterization of a stationary regular finite state Markov chain by its joint probability matrix $\Pi$, proposed a decomposition of $\Pi$ into its reversible part $X$ and irreversible part $L$, and suggested an index describing a certain kind of irreversibility in chains whose states are naturally ordered. Empirical examples illustrate the use of these quantities, for both directly observed and latent chains.

\footnotetext{
${ }^{6} \mu_{2}=0.01$ means an average $1 \%$ growth, at an annual rate with continuous compounding, in the moderate growth rate state
} 


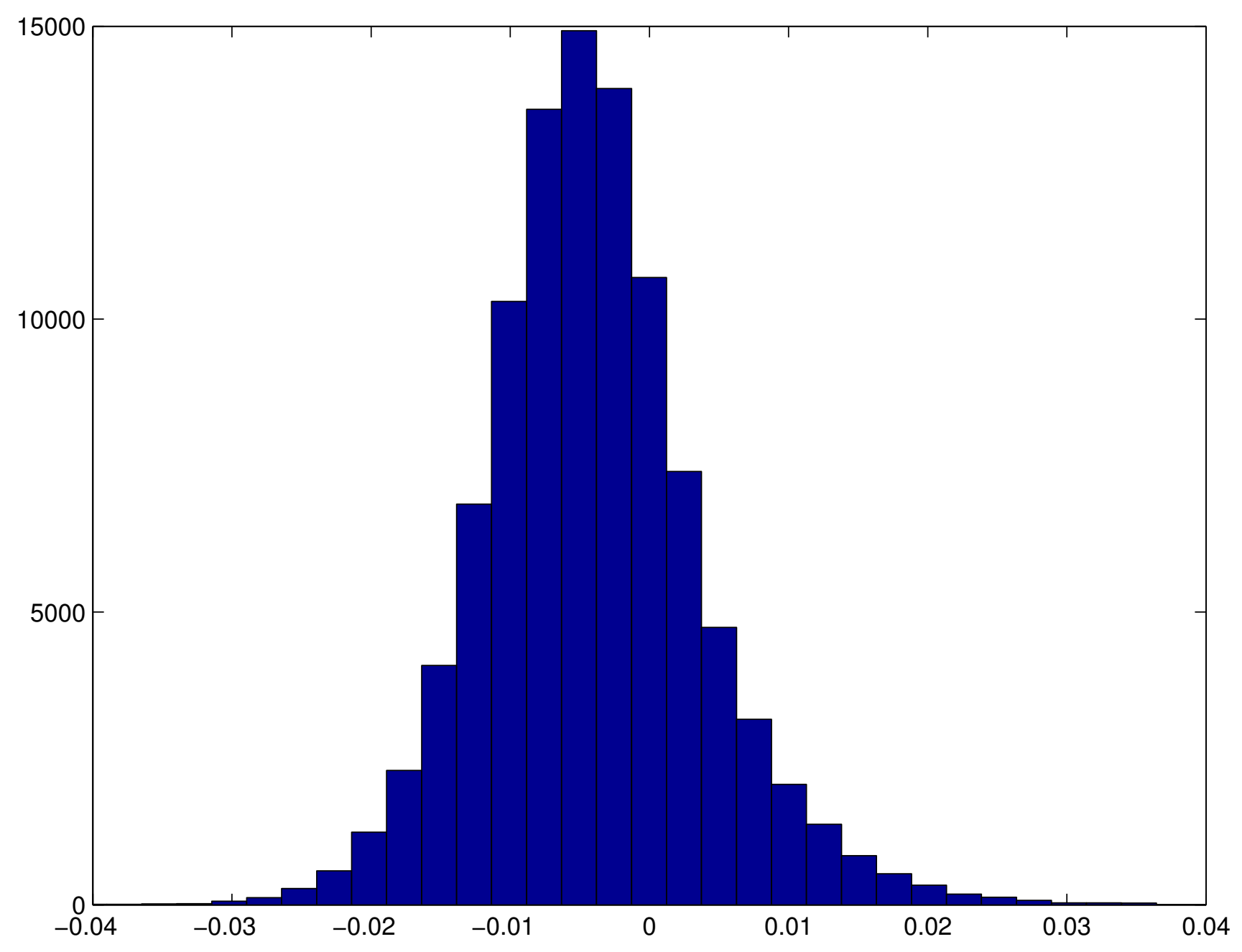

Figure 6: Posterior histogram of total circulation for the investment growth example 
In these examples, uncertainty about the dynamics of the Markov chain is expressed as a distribution over the Markov transition matrix $P$. Providing instead a marginal prior for $X$ and a conditional prior for $L \mid X$ would have many advantages:

1. A prior for $X$ is easy to elicit. For example, one can choose a Dirichlet distribution (or a mixture of such) for the following vector:

$$
\theta_{X} \equiv\left(X_{11}, 2 X_{12}, \ldots, 2 X_{1 m}, X_{22}, 2 X_{23}, \ldots, 2 X_{2 m}, \ldots, X_{m m}\right)
$$

2. The implied prior for $\pi$ depends only on the prior for $X$, (recall Result 3.2) and the fact that $\pi$ is a linear function of $X\left(\pi=\iota^{\prime} X\right)^{7}$ means that first and second moments of $\pi$ may be easy derived from the first two moments of $X$. The mean is particularly transparent: $E[\pi]=\iota^{\prime} E[X]$. Consider the difficulty of finding moments of $\pi$ given a prior on $P$.

3. Testing for reversibility of a stationary regular finite state Markov chain $\left\{s_{t}\right\}$ can be very disciplined. We can use a Bayes factor to compare 2 models for $\left\{s_{t}\right\}$ : one with a prior density $f(X)$ and $L=0$ with probability 1 and the other with the same density $f(X)$ and a prior density $f(L \mid X)$. Both models imply exactly the same prior distributions for $\pi$, the state persistence $P_{i i}$ in every state and the total flow $\Pi_{i j}+\Pi_{j i}$ between any two states (recall Result 3.2).

4. We can choose truncated priors for $L \mid X$ to impose restrictions such as the direction of total circulation $C$ or a common sign on the circulation through all interior states. We can also choose priors that are neutral about the direction of circulation without the extreme form of symmetry used in this paper.

However, elicitation of $L \mid X$ is not easy. Even with a flat prior, computing the normalization factor for $f(L \mid X)$, which we seem to need ${ }^{8}$ for posterior simulation since it depends on $X$, is no simple feat. A paper in preparation shows how we can elicit priors on $L \mid X$ and therefore realize the above advantages.

\footnotetext{
${ }^{7} \iota$ is an $m \times 1$ vector of ones

${ }^{8}$ We have a paper in preparation which shows that we do not in fact need this normalization factor.
} 


\section{References}

[1] Y.-T. Chen, R. Y. Chou, and C.-M. Kuan. Testing time reversibility without moment restrictions. Journal of Econometrics, 95:199-218, 2000 .

[2] Y.-T. Chen and C.-M. Kuan. Time irreversibility and EGARCH effect in U.S. stock index returns. In Proceedings of the International Conference on Modelling and Forecasting Financial Volatility. 2001.

[3] S. Darolles, J.-P. Florens, and C. Gourieroux. Kernel based nonlinear canonical analysis and time reversibility. Working Paper, Centre de Recherche en Economie et Statistique (CREST), 2000.

[4] A. Eckert. Retail price cycles and response asymmetry. Canadian Journal of Economics, 35, 2002.

[5] A. Eckert. Retail price cycles and presence of small firms. International Journal of Industrial Organization, 21, 2003.

[6] W. M. Fong. Time reversibility tests of volume-volatility dynamics for stock returns. Economics Letters, 81:39-45, 2003.

[7] John Geweke. Using simulation methods for Bayesian econometric models: Inference, development, and communication. Econometric Reviews, 18:1-126, 1999.

[8] James D. Hamilton. A new approach to the economic analysis of nonstationary time series and the business cycle. Econometrica, 57:357-84, 1989.

[9] M. J. Hinich and R. Rothman. A frequency domain test of time reversibility. Macroeconomic Dynamics, 2:72-88, 1998.

[10] M. Iosifescu. Finite Markov Processes and Their Applications. John Wiley \& Sons, 1980.

[11] J. Kemeny and J. L. Snell. Finite Markov Chains. D. Van Nostrand, New York, 1960. 
[12] E. Maskin and J. Tirole. A theory of dynamic oligopoly II: Price competition, kinked demand curves and Edgeworth cycles. Econometrica, 56:571-599, 1988.

[13] William J. McCausland. Using the BACC software for Bayesian inference. Journal of Computational Economics, 23:201-218, 2004.

[14] Michael Noel. Edgeworth price cycles, cost-based pricing and sticky pricing in retail gasoline markets. Unpublished Manuscript, University of California, San Diego, 2003.

[15] L. Peng and W. Xiong. Time to digest and volatility dynamics. Working Paper, Princeton University, 2003.

[16] J. B. Ramsey and P. Rothman. Time irreversibility and business cycle asymmetry. Journal of Money, Credit, and Banking, 28:3-20, 1996.

[17] P. M. Robinson. Consistent nonparametric entropy-based testing. Review of Economic Studies, 58, 1991.

[18] P. M. Rothman. The time reversibility test with application to financial data. In W. Semmler, editor, Business Cycles: Theory and Empirical Methods, pages 389-403. Kluwer Academic Publishers, Boston, 1994.

[19] C. A. Sims. Implications of rational inattention. Working Paper, Princeton University, 2001. 
Table 2: Posterior mean and standard deviation of the elements of $P, \pi$ and $\Pi$ in the gasoline markup example

\begin{tabular}{llllll}
\hline 0.4647 & 0.2048 & 0.1329 & 0.0660 & 0.0660 & 0.0656 \\
0.0828 & 0.5092 & 0.0411 & 0.2647 & 0.0613 & 0.0409 \\
0.0117 & 0.1811 & 0.5275 & 0.2329 & 0.0234 & 0.0234 \\
0.0085 & 0.0173 & 0.2704 & 0.5948 & 0.0923 & 0.0168 \\
0.0373 & 0.0768 & 0.1112 & 0.4794 & 0.2211 & 0.0741 \\
0.0719 & 0.0735 & 0.0723 & 0.1416 & 0.2851 & 0.3556 \\
\hline 0.1239 & 0.1010 & 0.0847 & 0.0619 & 0.0621 & 0.0616 \\
0.0392 & 0.0719 & 0.0283 & 0.0630 & 0.0339 & 0.0281 \\
0.0116 & 0.0413 & 0.0535 & 0.0453 & 0.0164 & 0.0162 \\
0.0083 & 0.0121 & 0.0404 & 0.0446 & 0.0263 & 0.0118 \\
0.0360 & 0.0511 & 0.0596 & 0.0947 & 0.0781 & 0.0496 \\
0.0669 & 0.0680 & 0.0675 & 0.0893 & 0.1165 & 0.1232 \\
\hline \hline 0.0508 & 0.1567 & 0.2747 & 0.3848 & 0.0868 & 0.0462 \\
\hline 0.0222 & 0.0322 & 0.0356 & 0.0408 & 0.0192 & 0.0181 \\
\hline \hline 0.0251 & 0.0099 & 0.0063 & 0.0031 & 0.0031 & 0.0032 \\
0.0128 & 0.0812 & 0.0063 & 0.0406 & 0.0094 & 0.0063 \\
0.0032 & 0.0491 & 0.1461 & 0.0636 & 0.0064 & 0.0063 \\
0.0032 & 0.0066 & 0.1032 & 0.2300 & 0.0353 & 0.0064 \\
0.0032 & 0.0066 & 0.0095 & 0.0412 & 0.0198 & 0.0064 \\
0.0032 & 0.0033 & 0.0032 & 0.0063 & 0.0127 & 0.0176 \\
\hline 0.0167 & 0.0058 & 0.0043 & 0.0030 & 0.0031 & 0.0032 \\
0.0064 & 0.0257 & 0.0045 & 0.0096 & 0.0052 & 0.0043 \\
0.0031 & 0.0102 & 0.0307 & 0.0132 & 0.0044 & 0.0044 \\
0.0031 & 0.0046 & 0.0135 & 0.0379 & 0.0102 & 0.0044 \\
0.0032 & 0.0046 & 0.0053 & 0.0107 & 0.0101 & 0.0047 \\
0.0032 & 0.0033 & 0.0032 & 0.0043 & 0.0063 & 0.0121 \\
\hline
\end{tabular}


Table 3: Posterior mean and standard deviation of the elements of $X$ and $L$ in the gasoline markup example

\begin{tabular}{rrrrrr}
\hline 0.0251 & 0.0114 & 0.0047 & 0.0032 & 0.0032 & 0.0032 \\
& 0.0812 & 0.0277 & 0.0236 & 0.0080 & 0.0048 \\
& & 0.1461 & 0.0834 & 0.0080 & 0.0048 \\
& & & 0.2300 & 0.0383 & 0.0063 \\
& & & & 0.0198 & 0.0096 \\
\hline 0.0167 & 0.0053 & 0.0029 & 0.0023 & 0.0024 & 0.0025 \\
& 0.0257 & 0.0061 & 0.0058 & 0.0038 & 0.0030 \\
& & 0.0307 & 0.0121 & 0.0037 & 0.0029 \\
& & & 0.0379 & 0.0094 & 0.0034 \\
& & & & 0.0101 & 0.0046 \\
\hline \hline 0 & -0.0015 & 0.0016 & -0.0000 & -0.0000 & -0.0000 \\
& 0 & -0.0214 & 0.0170 & 0.0014 & 0.0015 \\
& & 0 & -0.0198 & -0.0016 & 0.0016 \\
& & & 0 & -0.0029 & 0.0001 \\
& & & & 0 & -0.0031 \\
& 0.0030 & 0.0024 & 0.0020 & 0.0020 & 0.0020 \\
& 0 & 0.0050 & 0.0049 & 0.0031 & 0.0024 \\
& & 0 & 0.0056 & 0.0032 & 0.0025 \\
& & & 0 & 0.0046 & 0.0027 \\
& & & & 0 & 0.0030 \\
& & & & & 0 \\
\hline
\end{tabular}


Table 4: Posterior mean and standard deviation of circulation by state and total circulation, in the gasoline markup example

\begin{tabular}{ccc} 
State & Posterior Mean & Posterior Standard Deviation \\
\hline 2 & -0.0015 & 0.0030 \\
3 & -0.0198 & 0.0051 \\
4 & -0.0029 & 0.0048 \\
5 & -0.0031 & 0.0030 \\
\hline Total & -0.0272 & 0.0091 \\
\hline
\end{tabular}

Table 5: Posterior mean and standard deviation of the elements of $P, \pi, \Pi$, $X$ and $L$ in the investment growth example

\begin{tabular}{cccr}
\hline$P$ & $0.2953(0.1253)$ & $0.2864(0.1702)$ & $0.4182(0.1627)$ \\
& $0.0442(0.0297)$ & $0.9020(0.0882)$ & $0.0538(0.0805)$ \\
& $0.2104(0.1163)$ & $0.3061(0.1449)$ & $0.4835(0.1493)$ \\
\hline$\pi$ & $0.0876(0.0335)$ & $0.7676(0.1004)$ & $0.1448(0.0875)$ \\
\hline$\Pi$ & $0.0273(0.0196)$ & $0.0245(0.0171)$ & $0.0358(0.0184)$ \\
& $0.0328(0.0181)$ & $0.6989(0.1312)$ & $0.0359(0.0423)$ \\
& $0.0275(0.0180)$ & $0.0442(0.0372)$ & $0.0732(0.0618)$ \\
\hline$X$ & $0.0273(0.0196)$ & $0.0286(0.0158)$ & $0.0316(0.0164)$ \\
& & $0.6989(0.1312)$ & $0.0400(0.0391)$ \\
& & & $0.0732(0.0618)$ \\
\hline$L$ & $0(0)$ & $-0.0041(0.0079)$ & $0.0041(0.0079)$ \\
& & $0(0)$ & $-0.0041(0.0079)$ \\
& & & $0(0)$ \\
\hline
\end{tabular}

